\title{
Anabases
}

ANABASES Traditions et réceptions de l'Antiquité

$7 \mid 2008$

Varia

\section{Clarisse HERRENSCHMIDT, Les trois écritures. Langue, nombre, code}

\section{Corinne Bonnet}

\section{OpenEdition}

\section{Journals}

Édition électronique

URL : http://journals.openedition.org/anabases/2555

DOI : 10.4000/anabases. 2555

ISSN : 2256-9421

\section{Éditeur}

E.R.A.S.M.E.

\section{Édition imprimée}

Date de publication : 1 mars 2008

Pagination : 281-284

ISSN : 1774-4296

\section{Référence électronique}

Corinne Bonnet, «Clarisse heRRENSCHMIDT, Les trois écritures. Lanque, nombre, code», Anabases [En ligne],

7| 2008, mis en ligne le 01 décembre 2011, consulté le 20 septembre 2020. URL : http://

journals.openedition.org/anabases/2555; DOI : https://doi.org/10.4000/anabases.2555

Ce document a été généré automatiquement le 20 septembre 2020.

(c) Anabases 


\title{
Clarisse HERRENSCHMIDT, Les trois écritures. Langue, nombre, code
}

\author{
Corinne Bonnet
}

\section{RÉFÉRENCE}

Clarisse HERRENSCHMIDT, Les trois écritures. Langue, nombre, code, Bibliothèque des sciences humaines, Paris, Gallimard, 2007, 505 p.

29 euros / ISBN 978-2-07-076025-1.

1 « Nous vivons un grand chambardement sémiologique », un « moment particulier, celui de l'expansion mondiale d'une nouvelle écriture", souligne d'emblée Clarisse Herrenschmidt (p. VII). Si chacun s'accordera sur ce bilan, qui fait de l'écriture informatique et réticulaire une sorte de « révolution » contemporaine, rares sont ceux qui se sont efforcés d'analyser ce phénomène dans toute son épaisseur historique et d'en mesurer l'impact en termes de pratiques scientifiques et de modes de pensée. Telle est bien l'ambition du passionnant volume que propose Clarisse Herrenschmidt, articulé autour de trois modes d'écriture : celle des langues, celle des nombres, celle des codes informatiques.

2 L'analyse part d'un double postulat: celui du rapport intime et profond entre, d'une part, les choses du monde et les choses du langage - rapport intime au sein duquel l'écriture introduit une relative distanciation - et, d'autre part, entre les façons d'écrire et les façons de penser. Mais une fois ce postulat établi, reste à travailler la matière historique, car les régimes graphiques varient dans le temps, tout en véhiculant une part d'inertie culturelle, en rapport avec le poids de la tradition, mais aussi la résistance à l'uniformisation et à la désymbolisation. Bref, écritures et histoire font des nœuds, tissent une aventure millénaire que l'Auteur démêle patiemment, avec autant d'érudition que de finesse et d'originalité.

3 Le sujet, en effet, est d'une extrême complexité : technique et épistémologique à la fois, il est abordé ici dans la langue durée, du IVe millénaire av. J.-C. jusqu'au XXI ${ }^{\mathrm{e}}$ siècle, en 
trois parties reflétant la trilogie des écritures (langues, nombres, codes). La première partie s'enracine dans les premières expériences graphiques, en Mésopotamie. Quelle réflexion sur le rapport entre les choses et les signes écrits se trouve-t-elle de toile de fond, lorsqu'à Uruk apparaissent les premiers signes, pictogrammes, logogrammes, transcrivant probablement du sumérien, dont le fonctionnement est remarquablement élucidé. L'A. montre bien que la réflexivité (sous forme d'énoncés métalinguistiques minima) est d'emblée au cœur des signes d'écriture. L'introduction, par les Akkadiens, de signes syllabiques introduit un processus de décontextualisation, une autonomisation de l'écriture par rapport aux choses.

4 L'écriture alphabétique consonantique, comme chez les Phéniciens ou les Hébreux, repose, quant à elle, sur une autre logique : celle d'un système au sein duquel les mots se développent au départ de racines et où la lecture est fortement déterminée, voire conditionnée par l'environnement de l'énoncé. L'analyse conduite autour du nom de Dieu, YнWн (p. 46-52), indique très bien la manière dont l'énigme graphique est résolue ou non, en fonction du statut du mot. Le nom de үнwн fait partie de la création linguistique, mais il y jouit d'un statut particulier et supérieur, dans la mesure où l'énigme demeure.

Dans ce contexte, l'alphabet grec, avec sa notation transparente des consonnes et des voyelles, apparaît comme l'aboutissement du processus d'autonomisation des signes. Le mot disparaît devant la lettre, le texte devient un alignement régulier et uniforme de lettres toutes égales, un peu comme les citoyens, au sein d'une société statutairement égalitaire. Corps de lettres, corps civique: l'équation est suggestive. Ici aussi, l'approche concrète, autour du " je », des objets parlants et du dialogue socratiques est absolument passionnante (p. 52-60). On y apprend à regarder les signes d'écriture comme des faits sociaux. De nouveaux espaces d'interlocution émergent, une réflexion s'engage quant à l'origine des mots, leur étymologie, leur sens. Une réflexion qui, chez Platon, débouche sur une vision négative de l'écriture, mimesis imparfaite et décevante. Pour mieux mesurer l'originalité $\mathrm{du}$ " je » grec, autonome et critique, il faut revenir aux p. 41 à 46 , où l'A. propose une magnifique analyse du texte figurant sur un type de statue de Gudea de Lagash, une statue parlante qui s'adresse au dieu au nom du roi, en une époque où les mots, les choses, les noms, la langue formaient un tout solidaire.

6 Un détour par l'Iran élamite et par les maisons de Suse permet de remonter aux bullesenveloppes scellées, en forme de bouche (qui abrite la «langue»!), réceptacle d'un message (étourdissant le rapprochement avec le mythe d'Enmerkar, p. 78) et de calculi, attestés dès le VIIe millénaire. On en vient alors à s'interroger sur les rapports entre écriture et État, puis à suivre le parcours des écritures proto-élamites et élamites, cette dernière consignée par le biais d'un syllabaire cunéiforme akkadien, exemple de distension maximale entre la langue et l'écriture.

7 Analysant ensuite le fonctionnement des alphabets consonantiques, de l'écriture grecque et du cunéiforme vieux-perse, l'A. s'intéresse au rôle de l'acteur (ou sujet) linguistique (locuteur, lecteur), tantôt présent, tantôt absent, muet ou sonore, implicite ou explicite, conscient ou inconscient, inscrit ou non dans la dimension du temps. On perçoit ainsi des niveaux et des modalités de rationalité différents dans les écritures, ainsi que des articulations mobiles entre le visible et l'invisible, l'humain et le divin. Reste encore à percer les mystères d'une ultime dialectique, celle de l'oral et de l'écrit. La fixation de la langue par écrit contribue en effet à enrichir et approfondir les 
théories et les stratégies du langage. Un splendide détour par les terres mazdéennes, aux multiples écritures, que nous présente Clarisse Herrenschmidt, en fournit l'illustration, et même la démonstration. Ce sont les terres d'Ahura Mazda, « le lanceur et le propulseur des mots et des paroles ", au centre de l'échange, tout comme le roi qui, dans ses inscriptions, inlassablement "explique». L'écriture, en dépit de ses variantes, enracine et sacralise l'organisation sociale. La réforme de l'alphabet athénien en 403 confirme ce rôle de "gardien social» et de vecteur du "corps social» (p. 161-176). Une ultérieure mise en perspective de cette dimension de l'écriture résulte du cas de l'hébreu, langue morte après l'Antiquité, qui renaît de ses cendres après 1948.

Le lien indissoluble entre la condition humaine et l'écriture, évident dans toutes les phases de l'histoire, investit aussi la dimension mythique; la prise en compte de trois mythes, mésopotamien, biblique et grec, qui associent étroitement l'émergence du langage ou de l'écriture et la naissance de l'homme/la femme : « Le champ magnétique au complexe circuit de forces instauré fait de l'Homme et de l'écriture un couple de jumeaux.»

9 La seconde partie, que nous résumerons par la force des choses plus rapidement, est consacrée à l'écriture des nombres. Elle s'intéresse davantage à la vision du nombre qu'implique la graphie, notamment monétaire, plutôt qu'aux contextes de son apparition et de ses usages. Le point fort de l'argumentation est le nœud entre mathématiques et économie. L'A. s'arrête donc longuement sur l'écriture monétaire de la Grèce ionienne, en partant de l'Artémision d'Éphèse et en proposant une analyse approfondie du récit hérodotéen sur les offrandes de Crésus et son destin, en rapport avec Artémis, la déesse au masque. Ce qui conduit à mettre en évidence un lien entre les rites célébrés à Éphèse et l'invention de la monnaie.

10 Celle-ci donne naissance à une écriture monétaire proprement dite qui consiste initialement en des figures géométriques, objets d'un déchiffrement aux p. 280-289. Mais la géométrie et les nombres, en contexte monétaire, ont un rôle économique, social et même politique. Le discours aristotélicien sur la monnaie comme instrument de justice en témoigne très bien : c'est la cohérence du groupe qui prime, et non son enrichissement. Clarisse Herrenschmidt suit alors le développement de l'écriture des nombres dans l'Europe médiévale et moderne: crédit, lettre de change, contrats, comptabilité en partie double - si importante dans l'analyse de la rationalité moderne conduite par Max Weber, qui ne semble cependant pas évoqué dans ces pages - sont autant de facettes et d'étapes d'une évolution qui conduit au capitalisme. L'uniformisation du système métrique et surtout le développement des outils mathématiques contribuèrent alors à l'émergence d'une logique moderne. S'ouvre alors, au XIX siècle, "l'ère du chiffre", avec ses statistiques, ses courbes et ses graphiques. L'« écheveau sémiologique » du langage des nombres se dénoue en 1971, lorsque Richard Nixon sépara le dollar de l'or, transformant les monnaies en marchandises soumises aux spéculations, en signes écrits sur du papier ordinaire.

11 Troisième et dernier acte: la révolution graphique de l'informatique. Que signifie travailler avec un ordinateur, simulacre du graphique? Simulacre et simulation sont les mots clés du fonctionnement de l'écriture informatique. Mais aussi variété, avec toute une panoplie de langages informatiques. Ce sont donc bien nos pratiques de pensée, outre que de rédaction, qui sont profondément affectées par le recours à l'informatique. Ce sont aussi nos pratiques linguistiques avec l'irruption d'une acculturation anglo-américaine universelle. Avec cette troisième section, nous 
pénétrons dans l'univers du codage et du virtuel, un champ infini, puissance de transformation et de conservation plus éphémère qu'il n'y parait. Né par et pour le calcul, "l'outil informatique a ramené au même procédé d'écriture numérique les textes, les arts et les pratiques de l'image, les sons de la musique et de la voix vibrante » (p. 455). De cette uniformisation jaillit le réseau, « univers enchevêtré » de la Toile, qui émerge dans les années $90 \mathrm{du} \mathrm{xx}^{\mathrm{e}}$ siècle. Né dans les années tendues de la guerre froide, l'Internet, dont l'A. trace ici l'historique, devient le véhicule d'une communauté scientifique dont l'imaginaire produit une auto-représentation reposant sur la connectivité et l'élargissement des connaissances. "Présente et fragile», la Toile multiplie ses usages dans la société, mais génère souvent des liens faibles, ténus, superficiels, détournés ; elle produit aussi des effets paranoïdes et schizoïdes. Clarisse Herrenschmidt multiplie habilement les exemples et évite les analyses théoriques trop arides. On la suit aisément et volontiers dans les méandres du Net, sur les traces de personnages qui nous ressemblent plus ou moins. On visite la «bulle » de l'Internet, les chemins réticulaires d'un monde où l'économique, le financier imprime une marque forte, trop forte ; on pénètre au cœur du couple mythique homme-ordinateur localisé dans le cyberespace, avec tous ses paradoxes et ses tensions.

Qu'il me soit permis de clore par une image que j'emprunte à Clarisse Herrenschmidt et qui illustre très bien la finesse d'analyse et d'écriture d'un livre, qui n'est certes pas facile, mais qui est très riche et très stimulant, courageux, audacieux même par le champ chronologique et disciplinaire qu'il couvre. Qu'est-ce que le langage, en définitive ? Un filet où nous sommes comme un poisson dans l'eau (p. 64)...

\section{AUTEURS}

\section{CORINNE BONNET}

Université de Toulouse II-Le Mirail

corinne.bonnet@sfr.fr 